\title{
On Antiperiodic Boundary Value Problems for Higher-Order Fractional Differential Equations
}

\author{
Ahmed Alsaedi, Bashir Ahmad, and Afrah Assolami \\ Department of Mathematics, Faculty of Science, King Abdulaziz University, P.O. Box 80203, \\ Jeddah 21589, Saudi Arabia \\ Correspondence should be addressed to Ahmed Alsaedi, aalsaedi@hotmail.com \\ Received 18 May 2012; Accepted 1 July 2012 \\ Academic Editor: Dumitru Baleanu \\ Copyright (C) 2012 Ahmed Alsaedi et al. This is an open access article distributed under the \\ Creative Commons Attribution License, which permits unrestricted use, distribution, and \\ reproduction in any medium, provided the original work is properly cited.

\begin{abstract}
We study an antiperiodic boundary value problem of nonlinear fractional differential equations of order $q \in(4,5]$. Some existence results are obtained by applying some standard tools of fixed-point theory. We show that solutions for lower-order anti-periodic fractional boundary value problems follow from the solution of the problem at hand. Our results are new and generalize the existing results on anti-periodic fractional boundary value problems. The paper concludes with some illustrating examples.
\end{abstract}

\section{Introduction}

In the preceding years, there has been a great advancement in the study of fractional calculus. A variety of results on initial and boundary value problems of fractional order, ranging from the theoretical aspects of existence and uniqueness of solutions to the analytic and numerical methods for finding solutions, have appeared in the literature. It is mainly due to the extensive application of fractional differential equations in many engineering and scientific disciplines such as physics, chemistry, biology, economics, control theory, signal and image processing, biophysics, blood flow phenomena, aerodynamics, and fitting of experimental data [1-5]. For an updated account of mathematical tools for fractional models and methods of solutions for fractional differential equations, we refer the reader to a recent text [6] by Baleanu et al. Fractional derivatives are also regarded as an excellent tool for the description of memory and hereditary properties of various materials and processes [7]. These characteristics of the fractional derivatives make the fractional-order models more realistic and practical than the classical integer-order models. For more details and examples, see [8-20].

Antiperiodic boundary value problems occur in the mathematical modeling of a variety of physical processes and have received a considerable attention. Examples include 
antiperiodic trigonometric polynomials in the study of interpolation problems, antiperiodic wavelets, antiperiodic boundary conditions in physics, and so forth (for details, see [21] and the references therein). Some recent work on antiperiodic boundary value problems of fractional-order can be found in [21-27] and references therein.

In this paper, we consider an antiperiodic boundary value problems of fractional differential equations of order $q \in(4,5]$ given by

$$
\begin{aligned}
{ }^{c} D^{q} x(t) & =f(t, x(t)), \quad t \in[0, T], T>0,4<q \leq 5, \\
x(0) & =-x(T), \quad x^{\prime}(0)=-x^{\prime}(T), \quad x^{\prime \prime}(0)=-x^{\prime \prime}(T), \\
x^{\prime \prime \prime}(0) & =-x^{\prime \prime \prime}(T), \quad x^{(i v)}(0)=-x^{(i v)}(T),
\end{aligned}
$$

where ${ }^{c} D^{q}$ denotes the Caputo fractional derivative of order $q$ and $f$ is a given continuous function.

The main objective of the present work is to develop the existence theory for problem (1.1) and relate problem (1.1) with lower-order fractional antiperiodic boundary value problems. Our results are new and give further insight into the characteristics of fractionalorder antiperiodic boundary value problems.

\section{Preliminaries}

Definition 2.1 (see [4]). The Riemann-Liouville fractional integral of order $q$ for a continuous function $g$ is defined as

$$
I^{q} g(t)=\frac{1}{\Gamma(q)} \int_{0}^{t} \frac{g(s)}{(t-s)^{1-q}} d s, \quad q>0,
$$

provided the integral exists.

Definition 2.2 (see [4]). For at least $n$-times continuously differentiable function $g:[0, \infty) \rightarrow \mathbb{R}$, the Caputo derivative of fractional order $q$ is defined as

$$
{ }^{c} D^{q} g(t)=\frac{1}{\Gamma(n-q)} \int_{0}^{t}(t-s)^{n-q-1} g^{(n)}(s) d s, \quad n-1<q<n, n=[q]+1,
$$

where $[q]$ denotes the integer part of the real number $q$.

To study the nonlinear problem (1.1), we need the following lemma, which deals with a linear variant of problem (1.1). 
Lemma 2.3. For any $y \in C[0, T]$, the unique solution of the boundary value problem:

$$
\begin{aligned}
{ }^{c} D^{q} x(t) & =y(t), \quad t \in[0, T], 4<q \leq 5, \\
x(0) & =-x(T), \quad x^{\prime}(0)=-x^{\prime}(T), \quad x^{\prime \prime}(0)=-x^{\prime \prime}(T), \\
x^{\prime \prime \prime}(0) & =-x^{\prime \prime \prime}(T), \quad x^{(i v)}(0)=-x^{(i v)}(T)
\end{aligned}
$$

is

$$
x(t)=\int_{0}^{T} G(t, s) y(s) d s,
$$

where $G(t, s)$ is the Green's function given by

$$
G(t, s)= \begin{cases}\frac{2(t-s)^{q-1}-(T-s)^{q-1}}{2 \Gamma(q)}+\frac{(T-2 t)(T-s)^{q-2}}{4 \Gamma(q-1)}+\frac{t(T-t)(T-s)^{q-3}}{4 \Gamma(q-2)} & \\ +\frac{\left(6 t^{2} T-4 t^{3}-T^{3}\right)(T-s)^{q-4}}{48 \Gamma(q-3)}+\frac{\left(2 T t^{3}-t^{4}-t T^{3}\right)(T-s)^{q-5}}{48 \Gamma(q-4)}, & 0<s<t<T, \\ -\frac{(T-s)^{q-1}}{2 \Gamma(q)}+\frac{(T-2 t)(T-s)^{q-2}}{4 \Gamma(q-1)}+\frac{t(T-t)(T-s)^{q-3}}{4 \Gamma(q-2)} & 0<t<s<T . \\ +\frac{\left(6 t^{2} T-4 t^{3}-T^{3}\right)(T-s)^{q-4}}{48 \Gamma(q-3)}+\frac{\left(2 T t^{3}-t^{4}-t T^{3}\right)(T-s)^{q-5}}{48 \Gamma(q-4)}, & \end{cases}
$$

Proof. It is well known [4] that the solution of ${ }^{c} D^{q} x(t)=y(t)$ can be written as

$$
x(t)=\int_{0}^{t} \frac{(t-s)^{q-1}}{\Gamma(q)} y(s) d s-b_{o}-b_{1} t-b_{2} t^{2}-b_{3} t^{3}-b_{4} t^{4}
$$

where $b_{0}, b_{1}, b_{2}, b_{3}$, and $b_{4} \in \mathbb{R}$ are arbitrary constants. Using the boundary conditions for problem (2.3) in (2.6), we find that

$$
\begin{aligned}
b_{o}= & \frac{1}{2} \int_{0}^{T} \frac{(T-s)^{q-1}}{\Gamma(q)} y(s) d s-\frac{T}{4} \int_{0}^{T} \frac{(T-s)^{q-2}}{\Gamma(q-1)} y(s) d s \\
& +\frac{T^{3}}{48} \int_{0}^{T} \frac{(T-s)^{q-4}}{\Gamma(q-3)} y(s) d s,
\end{aligned}
$$




$$
\begin{aligned}
& b_{1}=\frac{1}{2} \int_{0}^{T} \frac{(T-s)^{q-2}}{\Gamma(q-1)} y(s) d s-\frac{T}{4} \int_{0}^{T} \frac{(T-s)^{q-3}}{\Gamma(q-2)} y(s) d s+\frac{T^{3}}{48} \int_{0}^{T} \frac{(T-s)^{q-5}}{\Gamma(q-4)} y(s) d s, \\
& b_{2}=\frac{1}{4} \int_{0}^{T} \frac{(T-s)^{q-3}}{\Gamma(q-2)} y(s) d s-\frac{T}{8} \int_{0}^{T} \frac{(T-s)^{q-4}}{\Gamma(q-3)} y(s) d s, \\
& b_{3}=\frac{1}{12} \int_{0}^{T} \frac{(T-s)^{q-4}}{\Gamma(q-3)} y(s) d s-\frac{T}{24} \int_{0}^{T} \frac{(T-s)^{q-5}}{\Gamma(q-4)} y(s) d s, \\
& b_{4}=\frac{1}{48} \int_{0}^{T} \frac{(T-s)^{q-5}}{\Gamma(q-4)} y(s) d s .
\end{aligned}
$$

Substituting the values of $b_{o}, b_{1}, b_{2}, b_{3}$, and $b_{4}$ in (2.6), we obtain

$$
\begin{aligned}
x(t)= & \int_{0}^{t} \frac{(t-s)^{q-1}}{\Gamma(q)} y(s) d s-\frac{1}{2} \int_{0}^{T} \frac{(T-s)^{q-1}}{\Gamma(q)} y(s) d s \\
& +\frac{(T-2 t)}{4} \int_{0}^{T} \frac{(T-s)^{q-2}}{\Gamma(q-1)} y(s) d s+\frac{t(T-t)}{4} \int_{0}^{T} \frac{(T-s)^{q-3}}{\Gamma(q-2)} y(s) d s \\
& +\frac{\left(6 t^{2} T-4 t^{3}-T^{3}\right)}{48} \int_{0}^{T} \frac{(T-s)^{q-4}}{\Gamma(q-3)} y(s) d s+\frac{\left(2 T t^{3}-t^{4}-t T^{3}\right)}{48} \int_{0}^{T} \frac{(T-s)^{q-5}}{\Gamma(q-4)} y(s) d s \\
= & \int_{0}^{T} G(t, s) y(s) d s,
\end{aligned}
$$

where $G(t, s)$ is given by (2.5). This completes the proof.

\subsection{Relationship with Lower-Order Problems}

We observe that the first term in expressions for $G(t, s)$ given by (2.5) corresponds to the Green's function for the problem:

$$
\begin{gathered}
{ }^{c} D^{q} x(t)=f(t, x(t)), \quad t \in[0, T], T>0,0<q \leq 1, \\
x(0)=-x(T) ;
\end{gathered}
$$

the first two terms in (2.5) form Green's function for the problem [21]:

$$
\begin{gathered}
{ }^{c} D^{q} x(t)=f(t, x(t)), \quad t \in[0, T], T>0,1<q \leq 2, \\
x(0)=-x(T), \quad x^{\prime}(0)=-x^{\prime}(T) ;
\end{gathered}
$$


the first three terms in (2.5) give the Green's function for the problem [22]:

$$
\begin{gathered}
{ }^{c} D^{q} x(t)=f(t, x(t)), \quad t \in[0, T], T>0,2<q \leq 3, \\
x(0)=-x(T), \quad x^{\prime}(0)=-x^{\prime}(T), \quad x^{\prime \prime}(0)=-x^{\prime \prime}(T) ;
\end{gathered}
$$

while the first four terms in (2.5) yield the Green's function for the antiperiodic problem [23]:

$$
\begin{gathered}
{ }^{c} D^{q} x(t)=f(t, x(t)), \quad t \in[0, T], T>0,3<q \leq 4, \\
x(0)=-x(T), \quad x^{\prime}(0)=-x^{\prime}(T), \quad x^{\prime \prime}(0)=-x^{\prime \prime}(T), \quad x^{\prime \prime \prime}(0)=-x^{\prime \prime \prime}(T) .
\end{gathered}
$$

From the above deductions, it can easily be concluded that Green's function (2.5) for an antiperiodic boundary value problem of fractional order $q \in(4,5]$ contains Green's function (or solution) for lower-order fractional antiperiodic problems. We can further interpret that the last term in expressions for Green's function (2.5) arises due to consideration of the order $q \in(4,5]$, whereas the remaining terms correspond to the lower-order problems. This observation gives a useful insight into the study of antiperiodic fractional boundary value problems that a unit-increase in the fractional order of the problem gives rise to a new term in expressions for Green's function, preserving the terms corresponding to lower-order antiperiodic problems. In other words, one can say that Green's function (or solution) for a higher-order antiperiodic fractional boundary value problem inherits all the characteristics of lower-order fractional antiperiodic problems. Hence, our results generalize the existing results on antiperiodic fractional boundary value problems ([21-23]).

\section{Existence Results}

Let $\mathcal{\varepsilon}:=C([0, T], \mathbb{R})$ denotes the Banach space of all continuous functions defined on $[0, T] \times \mathbb{R}$ endowed with a topology of uniform convergence with the norm $\|x\|=\sup _{t \in[0, T]}|x(t)|$. [28].

To prove the existence results for problem (1.1), we need the following known results

Theorem 3.1. Let $X$ be a Banach space. Assume that $T: X \rightarrow X$ is completely continuous operator and the set

$$
V=\{u \in X \mid u=\mu T u, 0<\mu<1\}
$$

is bounded. Then $T$ has a fixed point in X.

Theorem 3.2. Let $X$ be a Banach space. Assume that $\Omega$ is an open-bounded subset of $X$ with $\theta \in \Omega$ and let $T: \bar{\Omega} \rightarrow X$ be a completely continuous operator such that

$$
\|T u\| \leq\|u\|, \quad \forall u \in \partial \Omega .
$$

Then $T$ has a fixed point in $\bar{\Omega}$. 
By Lemma 2.3, we define an operator $\mathcal{U}: \varepsilon \rightarrow \varepsilon$ as

$$
\begin{aligned}
(\mathcal{U} x)(t)= & \int_{0}^{t} \frac{(t-s)^{q-1}}{\Gamma(q)} f(s, x(s)) d s-\frac{1}{2} \int_{0}^{T} \frac{(T-s)^{q-1}}{\Gamma(q)} f(s, x(s)) d s \\
& +\frac{(T-2 t)}{4} \int_{0}^{T} \frac{(T-s)^{q-2}}{\Gamma(q-1)} f(s, x(s)) d s+\frac{t(T-t)}{4} \int_{0}^{T} \frac{(T-s)^{q-3}}{\Gamma(q-2)} f(s, x(s)) d s \\
& +\frac{\left(6 t^{2} T-4 t^{3}-T^{3}\right)}{48} \int_{0}^{T} \frac{(T-s)^{q-4}}{\Gamma(q-3)} f(s, x(s)) d s \\
& +\frac{\left(2 T t^{3}-t^{4}-t T^{3}\right)}{48} \int_{0}^{T} \frac{(T-s)^{q-5}}{\Gamma(q-4)} f(s, x(s)) d s, \quad t \in[0, T] .
\end{aligned}
$$

Observe that the problem (1.1) has a solution if and only if the operator equation $\mathcal{U} x=$ $x$ has a fixed point.

Theorem 3.3. Assume that there exists a positive constant $L_{1}$ such that $|f(t, x)| \leq L_{1}$ for $t \in$ $[0, T], x \in \mathbb{R}$. Then the problem (1.1) has at least one solution.

Proof. First of all, we show that the operator $\mathcal{U}$ is completely continuous. Note that the operator $U$ is continuous in view of the continuity of $f$. Let $B \subset \mathcal{E}$ be a bounded set. By the assumption that $|f(t, x)| \leq L_{1}$, for $x \in B$, we have

$$
\begin{aligned}
|(\mathcal{U} x)(t)| \leq & \int_{0}^{t} \frac{(t-s)^{q-1}}{\Gamma(q)}|f(s, x(s))| d s+\frac{1}{2} \int_{0}^{T} \frac{(T-s)^{q-1}}{\Gamma(q)}|f(s, x(s))| d s \\
& +\frac{1}{4}|T-2 t| \int_{0}^{T} \frac{(T-s)^{q-2}}{\Gamma(q-1)}|f(s, x(s))| d s \\
& +\frac{1}{4}|t(T-t)| \int_{0}^{T} \frac{(T-s)^{q-3}}{\Gamma(q-2)}|f(s, x(s))| d s \\
& +\frac{\left|6 t^{2} T-4 t^{3}-T^{3}\right|}{48} \int_{0}^{T} \frac{(T-s)^{q-4}}{\Gamma(q-3)}|f(s, x(s))| d s \\
& +\frac{\left|2 T t^{3}-t^{4}-t T^{3}\right|}{48} \int_{0}^{T} \frac{(T-s)^{q-5}}{\Gamma(q-4)}|f(s, x(s))| d s \\
\leq & L_{1}\left[\frac{1}{\Gamma(q)} \int_{0}^{t}(t-s)^{q-1} d s+\frac{1}{2 \Gamma(q)} \int_{0}^{T}(T-s)^{q-1} d s\right. \\
& +\frac{|T-2 t|}{4 \Gamma(q-1)} \int_{0}^{T}(T-s)^{q-2} d s+\frac{|t(T-t)|}{4 \Gamma(q-2)} \int_{0}^{T}(T-s)^{q-3} d s
\end{aligned}
$$


Abstract and Applied Analysis

$$
\begin{gathered}
\left.+\frac{\left|6 t^{2} T-4 t^{3}-T^{3}\right|}{48 \Gamma(q-3)} \int_{0}^{T}(T-s)^{q-4} d s+\frac{\left|2 T t^{3}-t^{4}-t T^{3}\right|}{48 \Gamma(q-4)} \int_{0}^{T}(T-s)^{q-5} d s\right] \\
\leq L_{1}\left[\frac{T^{q}}{2 \Gamma(q+1)}\left(3+\frac{q}{2}+\frac{q(q-1)\left(5 q^{2}-9 q+46\right)}{384}\right)\right]=L_{2},
\end{gathered}
$$

which implies that $\|(\mathcal{U} x)\| \leq L_{2}$. Further, we find that

$$
\begin{aligned}
\left|(\mathcal{U} x)^{\prime}(t)\right|= & \int_{0}^{t} \frac{(t-s)^{q-2}}{\Gamma(q-1)}|f(s, x(s))| d s+\frac{1}{2} \int_{0}^{T} \frac{(T-s)^{q-2}}{\Gamma(q-1)}|f(s, x(s))| d s \\
& +\frac{|T-2 t|}{4} \int_{0}^{T} \frac{(T-s)^{q-3}}{\Gamma(q-2)}|f(s, x(s))| d s+\frac{|t(T-t)|}{4} \int_{0}^{T} \frac{(T-s)^{q-4}}{\Gamma(q-3)}|f(s, x(s))| d s \\
& +\frac{\left|6 T t^{2}-4 t^{3}-T^{3}\right|}{48} \int_{0}^{T} \frac{(T-s)^{q-5}}{\Gamma(q-4)}|f(s, x(s))| d s \\
\leq & L_{1}\left[\int_{0}^{t} \frac{(t-s)^{q-2}}{\Gamma(q-1)} d s+\frac{1}{2} \int_{0}^{T} \frac{(T-s)^{q-2}}{\Gamma(q-1)} d s\right. \\
& +\frac{|T-2 t|}{4} \int_{0}^{T} \frac{(T-s)^{q-3}}{\Gamma(q-2)} d s+\frac{|t(T-t)|}{4} \int_{0}^{T} \frac{(T-s)^{q-4}}{\Gamma(q-3)} d s \\
\leq & L_{1}\left[\frac{\left|6 T t^{2}-4 t^{3}-T^{3}\right|}{48} \int_{0}^{T} \frac{(T-s)^{q-5}}{\Gamma(q-4)}\right] \\
& {\left.\left[3+\frac{(q-1)\left(q^{2}-2 q+12\right)}{24}\right)\right]=L_{3} . }
\end{aligned}
$$

Hence, for $t_{1}, t_{2} \in[0, T]$, we have

$$
\left|(\mathcal{U} x)\left(t_{2}\right)-(\mathcal{U} x)\left(t_{1}\right)\right| \leq \int_{t_{1}}^{t_{2}}\left|(\mathcal{U} x)^{\prime}(s)\right| d s \leq L_{3}\left(t_{2}-t_{1}\right) .
$$

This implies that $\mathcal{U}$ is equicontinuous on $[0, T]$. Thus, by the Arzela-Ascoli theorem, the operator $u: \varepsilon \rightarrow \varepsilon$ is completely continuous.

Next, we consider the set

$$
V=\{x \in \mathcal{E} \mid x=\mu \varkappa x, 0<\mu<1\}
$$


and show that the set $V$ is bounded. Let $x \in V$, then $x=\mu \varkappa x, 0<\mu<1$. For any $t \in[0, T]$, we have

$$
\begin{aligned}
& x(t)=\int_{0}^{t} \frac{(t-s)^{q-1}}{\Gamma(q)} f(s, x(s)) d s-\frac{1}{2} \int_{0}^{T} \frac{(T-s)^{q-1}}{\Gamma(q)} f(s, x(s)) d s \\
& +\frac{(T-2 t)}{4} \int_{0}^{T} \frac{(T-s)^{q-2}}{\Gamma(q-1)} f(s, x(s)) d s+\frac{t(T-t)}{4} \int_{0}^{T} \frac{(T-s)^{q-3}}{\Gamma(q-2)} f(s, x(s)) d s \\
& +\frac{\left(6 t^{2} T-4 t^{3}-T^{3}\right)}{48} \int_{0}^{T} \frac{(T-s)^{q-4}}{\Gamma(q-3)} f(s, x(s)) d s \\
& +\frac{\left(2 T t^{3}-t^{4}-t T^{3}\right)}{48} \int_{0}^{T} \frac{(T-s)^{q-5}}{\Gamma(q-4)} f(s, x(s)) d s, \\
& |x(t)|=\mu|(\mathcal{U} x)(t)| \leq \int_{0}^{t} \frac{(t-s)^{q-1}}{\Gamma(q)}|f(s, x(s))| d s+\frac{1}{2} \int_{0}^{T} \frac{(T-s)^{q-1}}{\Gamma(q)}|f(s, x(s))| d s \\
& +\frac{|T-2 t|}{4} \int_{0}^{T} \frac{(T-s)^{q-2}}{\Gamma(q-1)}|f(s, x(s))| d s \\
& +\frac{|t(T-t)|}{4} \int_{0}^{T} \frac{(T-s)^{q-3}}{\Gamma(q-2)}|f(s, x(s))| d s \\
& +\frac{\left|6 t^{2} T-4 t^{3}-T^{3}\right|}{48} \int_{0}^{T} \frac{(T-s)^{q-4}}{\Gamma(q-3)}|f(s, x(s))| d s \\
& +\frac{\left|2 T t^{3}-t^{4}-t T^{3}\right|}{48} \int_{0}^{T} \frac{(T-s)^{q-5}}{\Gamma(q-4)}|f(s, x(s))| d s \\
& \leq L_{1}\left[\frac{1}{\Gamma(q)} \int_{0}^{t}(t-s)^{q-1} d s+\frac{1}{2 \Gamma(q)} \int_{0}^{T}(T-s)^{q-1} d s\right. \\
& +\frac{|T-2 t|}{4 \Gamma(q-1)} \int_{0}^{T}(T-s)^{q-2} d s+\frac{|t(T-t)|}{4 \Gamma(q-2)} \int_{0}^{T}(T-s)^{q-3} d s \\
& +\frac{\left|6 t^{2} T-4 t^{3}-T^{3}\right|}{48 \Gamma(q-3)} \int_{0}^{T}(T-s)^{q-4} \Gamma(q-3) d s \\
& \left.+\frac{\left|2 T t^{3}-t^{4}-t T^{3}\right|}{48 \Gamma(q-4)} \int_{0}^{T}(T-s)^{q-5} d s\right] \\
& \leq \max _{t \in[0, T]}\left\{\frac{2\left|t^{q}\right|+T^{q}}{2 \Gamma(q+1)}+\frac{|T-2 t| T^{q-1}}{4 \Gamma(q)}+\frac{|t(T-t)| T^{q-2}}{4 \Gamma(q-1)}+\frac{\left|6 t^{2} T-4 t^{3}-T^{3}\right| T^{q-3}}{48 \Gamma(q-2)}\right. \\
& \left.+\frac{\left|2 T t^{3}-t^{4}-t T^{3}\right| T^{q-4}}{48 \Gamma(q-3)}\right\} L_{1}
\end{aligned}
$$


Thus, $\|x\| \leq M_{1}$ for any $t \in[0, T]$. So, the set $V$ is bounded. Thus, by the conclusion of Theorem 3.1, the operator $\mathfrak{U}$ has at least one fixed point, which implies that (1.1) has at least one solution.

Theorem 3.4. Let there exist a small positive number $\tau$ such that $|f(t, x)| \leq \delta|x|$ for $0<|x|<\tau$, where $\delta>0$ satisfies the condition

$$
\begin{gathered}
\max _{t \in[0, T]}\left\{\frac{2\left|t^{q}\right|+T^{q}}{2 \Gamma(q+1)}+\frac{|T-2 t| T^{q-1}}{4 \Gamma(q)}+\frac{|t(T-t)| T^{q-2}}{4 \Gamma(q-1)}+\frac{\left|6 t^{2} T-4 t^{3}-T^{3}\right| T^{q-3}}{48 \Gamma(q-2)}\right. \\
\left.+\frac{\left|2 T t^{3}-t^{4}-t T^{3}\right| T^{q-4}}{48 \Gamma(q-3)}\right\} \delta \leq 1 .
\end{gathered}
$$

Then the problem (1.1) has at least one solution.

Proof. Let us define $\boldsymbol{B}_{\tau}=\{x \in \mathcal{E} \mid\|x\|<\tau\}$ and take $x \in \mathcal{E}$ such that $\|x\|=\tau$, that is, $x \in \partial \boldsymbol{B}_{\tau}$. As before, it can be shown that $\mathcal{U}$ is completely continuous and

$$
\begin{gathered}
\|\mathcal{U} x\| \leq \max _{t \in[0, T]}\left\{\frac{2\left|t^{q}\right|+T^{q}}{2 \Gamma(q+1)}+\frac{|T-2 t| T^{q-1}}{4 \Gamma(q)}+\frac{|t(T-t)| T^{q-2}}{4 \Gamma(q-1)}+\frac{\left|6 t^{2} T-4 t^{3}-T^{3}\right| T^{q-3}}{48 \Gamma(q-2)}\right. \\
\left.+\frac{\left|2 T t^{3}-t^{4}-t T^{3}\right| T^{q-4}}{48 \Gamma(q-3)}\right\} \delta\|x\|,
\end{gathered}
$$

which in view of (3.10) yields $\|\mathcal{U} x\| \leq\|x\|, x \in \partial \mathfrak{B}_{\tau}$. Therefore, by Theorem 3.2, the operator $\mathcal{U}$ has at least one fixed point, which in turn implies that the problem (1.1) has at least one solution.

Our next existence result is based on Krasnoselskii's fixed point theorem [29].

Theorem 3.5. Let $M$ be a closed convex and nonempty subset of a Banach space X. Let $A$ and $B$ be the operators such that (i) $A x+B y \in M$ whenever $x, y \in M$; (ii) $A$ is compact and continuous; (iii) $B$ is a contraction mapping. Then there exists $z \in M$ such that $z=A z+B z$.

Theorem 3.6. Let $f:[0, T] \times \mathbb{R} \rightarrow \mathbb{R}$ be a jointly continuous function. Further, we assume that

$\left(\mathrm{A}_{1}\right)|f(t, x)-f(t, y)| \leq L|x-y|$, for all $t \in[0, T], x, y \in \mathbb{R}$

$\left(\mathrm{A}_{2}\right)|f(t, x)| \leq \mu(t)$, for all $(t, x) \in[0, T] \times \mathbb{R}$, and $\mu \in \mathbb{C}\left([0, T], R^{+}\right)$.

Then the problem (1.1) has at least one solution on $[0, T]$ if

$$
\frac{L T^{q}}{2 \Gamma(q+1)}\left(1+\frac{q}{2}+\frac{q(q-1)}{8}+\frac{q(q-1)(q-2)}{24}+\frac{5 q(q-1)(q-2)(q-3)}{384}\right)<1
$$


Proof. Letting $\sup _{t \in[0,1]}|\mu(t)|=\|\mu\|$, we fix

$$
\bar{r} \geq \frac{\|\mu\| T^{q}}{2 \Gamma(q+1)}\left(3+\frac{q}{2}+\frac{q(q-1)\left(5 q^{2}-9 q+46\right)}{384}\right)
$$

and consider $\boldsymbol{B}_{\bar{r}}=\{x \in \mathcal{E}:\|x\| \leq \bar{r}\}$. We define the operators $\mathcal{U}_{1}$ and $\mathcal{U}_{2}$ on $\boldsymbol{B}_{\bar{r}}$ as

$$
\begin{aligned}
&\left(\mathfrak{U}_{1} x\right)(t)=\int_{0}^{t} \frac{(t-s)^{q-1}}{\Gamma(q)} f(s, x(s)) d s \\
&\left(\mathcal{U}_{2} x\right)(t)=-\frac{1}{2} \int_{0}^{T} \frac{(T-s)^{q-1}}{\Gamma(q)} f(s, x(s)) d s+\frac{1}{4}(T-2 t) \int_{0}^{T} \frac{(T-s)^{q-2}}{\Gamma(q-1)} f(s, x(s)) d s \\
&+\frac{1}{4}(t(T-t)) \int_{0}^{T} \frac{(T-s)^{q-3}}{\Gamma(q-2)} f(s, x(s)) d s \\
&+\frac{\left(6 t^{2} T-4 t^{3}-T^{3}\right)}{48} \int_{0}^{T} \frac{(T-s)^{q-4}}{\Gamma(q-3)} f(s, x(s)) d s \\
&+\frac{\left(2 T t^{3}-t^{4}-t T^{3}\right)}{48} \int_{0}^{T} \frac{(T-s)^{q-5}}{\Gamma(q-4)} f(s, x(s)) d s .
\end{aligned}
$$

For $x, y \in B_{\bar{r}}$, we find that

$$
\left\|\mathfrak{u}_{1} x+\mathfrak{u}_{2} y\right\| \leq \frac{\|\mu\| T^{q}}{2 \Gamma(q+1)}\left(3+\frac{q}{2}+\frac{q(q-1)\left(5 q^{2}-9 q+46\right)}{384}\right) \leq r .
$$

Thus, $\mathfrak{U}_{1} x+\mathfrak{U}_{2} y \in \mathbb{B}_{\bar{r}}$. It follows from the assumption $\left(\mathrm{A}_{1}\right)$ that $\mathfrak{U}_{2}$ is a contraction mapping for

$$
\frac{L T^{q}}{2 \Gamma(q+1)}\left(1+\frac{q}{2}+\frac{q(q-1)}{8}+\frac{q(q-1)(q-2)}{24}+\frac{5 q(q-1)(q-2)(q-3)}{384}\right)<1
$$

Continuity of $f$ implies that the operator $\boldsymbol{U}_{1}$ is continuous. Also, $\boldsymbol{U}_{1}$ is uniformly bounded on $B_{\bar{r}}$ as

$$
\left\|\mathcal{U}_{1} x\right\| \leq \frac{\|\mu\| T^{q}}{\Gamma(q+1)}
$$

Now we prove the compactness of the operator $\mathfrak{U}_{1}$. In view of $\left(\mathrm{A}_{1}\right)$, we define

$$
\sup _{(t, x) \in[0, T] \times \bar{B}_{\bar{r}}}|f(t, x)|=f_{m}<\infty,
$$


and consequently, for $t_{1}, t_{2} \in[0, T]$ with $t_{1}<t_{2}$, we have

$$
\begin{gathered}
\left|\left(\mathfrak{U}_{1} x\right)\left(t_{2}\right)-\left(\mathfrak{U}_{1} x\right)\left(t_{1}\right)\right| \leq \frac{f_{m}}{\Gamma(q)} \mid \int_{0}^{t_{1}}\left[\left(t_{2}-s\right)^{q-1}-\left(t_{1}-s\right)^{q-1}\right] d s \\
+\int_{t_{1}}^{t_{2}}\left(t_{2}-s\right)^{q-1} d s \mid
\end{gathered}
$$

which is independent of $x$ and tends to zero as $t_{2}-t_{1} \rightarrow 0$. So $\mathfrak{U}_{1}$ is relatively compact on $\boldsymbol{B}_{\bar{r}}$. Hence, By the Arzela-Ascoli theorem, $\boldsymbol{U}_{1}$ is compact on $\boldsymbol{B}_{\bar{r}}$. Thus all the assumptions of Theorem 3.5 are satisfied. Therefore, the conclusion of Theorem 3.5 applies and the antiperiodic fractional boundary value problem (1.1) has at least one solution on $[0, T]$. This completes the proof.

Theorem 3.7. Assume that $f:[0, T] \times \mathbb{R} \rightarrow \mathbb{R}$ is a jointly continuous function satisfying the condition

$$
|f(t, x)-f(t, y)| \leq L|x-y|, \quad \forall t \in[0, T], x, y \in \mathbb{R}
$$

with

$$
\begin{gathered}
L \Delta<1, \\
\Delta=\frac{T^{q}}{2 \Gamma(q+1)}\left(3+\frac{q}{2}+\frac{q(q-1)\left(5 q^{2}-9 q+46\right)}{384}\right) .
\end{gathered}
$$

Then the antiperiodic boundary value problem (1.1) has a unique solution.

Proof. Let us define $\sup _{t \in[0, T]}|f(t, 0)|=M$ and select $r_{\kappa} \geq M \Delta /(1-\kappa)$ where $L \Delta \leq \kappa<1$. Then we show that $U B_{r_{\kappa}} \subset B_{r_{\kappa}}$, where $B_{r_{\kappa}}=\left\{x \in \mathcal{E}:\|x\| \leq r_{\kappa}\right\}$. For $x \in B_{r_{\kappa}}$, we have

$$
\begin{aligned}
\|(\mathcal{U} x)\| \leq \max _{t \in[0, T]}\left\{\int_{0}^{t} \frac{(t-s)^{q-1}}{\Gamma(q)}(|f(s, x(s))-f(s, 0)|+|f(s, 0)|) d s\right. \\
\quad+\frac{1}{2} \int_{0}^{T} \frac{(T-s)^{q-1}}{\Gamma(q)}(|f(s, x(s))-f(s, 0)|+|f(s, 0)|) d s \\
\quad+\frac{1}{4}|T-2 t| \int_{0}^{T} \frac{(T-s)^{q-2}}{\Gamma(q-1)}(|f(s, x(s))-f(s, 0)|+|f(s, 0)|) d s
\end{aligned}
$$




$$
\begin{gathered}
+\frac{1}{4}|t(T-t)| \int_{0}^{T} \frac{(T-s)^{q-3}}{\Gamma(q-2)}(|f(s, x(s))-f(s, 0)|+|f(s, 0)|) d s \\
+\frac{1}{48}\left|6 t^{2} T-4 t^{3}-T^{3}\right| \int_{0}^{T} \frac{(T-s)^{q-4}}{\Gamma(q-3)}(|f(s, x(s))-f(s, 0)|+|f(s, 0)|) d s \\
\left.+\frac{\left|2 T t^{3}-t^{4}-t T^{3}\right|}{48} \int_{0}^{T} \frac{(T-s)^{q-5}}{\Gamma(q-4)}(|f(s, x(s))-f(s, 0)|+|f(s, 0)|) d s\right\} \\
\leq\left(L r_{\kappa}+M\right) \max _{t \in[0, T]}\left\{\frac{1}{\Gamma(q)} \int_{0}^{t}(t-s)^{q-1} d s+\frac{1}{2 \Gamma(q)} \int_{0}^{T}(T-s)^{q-1} d s\right. \\
+\frac{|T-2 t|}{4 \Gamma(q-1)} \int_{0}^{T}(T-s)^{q-2} d s+\frac{|t(T-t)|}{4 \Gamma(q-2)} \int_{0}^{T}(T-s)^{q-3} d s \\
\quad+\frac{1}{48 \Gamma(q-3)}\left|6 t^{2} T-4 t^{3}-T^{3}\right| \int_{0}^{T}(T-s)^{q-4} d s \\
\leq\left(L r_{\kappa}+M\right)\left[\frac{T^{q}}{2 \Gamma(q+1)}\left(3+\frac{q}{2}+\frac{q(q-1)\left(5 q^{2}-9 q+46\right)}{384}\right)\right] \\
=\left(L r_{\kappa}+M\right) \Delta \leq \kappa r_{\kappa}+M \Delta \leq r_{\kappa},
\end{gathered}
$$

where (3.22) is used. Now, for $x, y \in \mathcal{E}$, we obtain

$$
\begin{aligned}
\|(\mathcal{U} x)-(\mathcal{U} y)\| \\
\leq \max _{t \in[0, T]}\left\{\int_{0}^{t} \frac{(t-s)^{q-1}}{\Gamma(q)}\|f(s, x(s))-f(s, y(s))\| d s\right. \\
+\frac{1}{2} \int_{0}^{T} \frac{(T-s)^{q-1}}{\Gamma(q)}\|f(s, x(s))-f(s, y(s))\| d s \\
+\frac{1}{4}|T-2 t| \int_{0}^{T} \frac{(T-s)^{q-2}}{\Gamma(q-1)}\|f(s, x(s))-f(s, y(s))\| d s \\
+\frac{1}{4}|t(T-t)| \int_{0}^{T} \frac{(T-s)^{q-3}}{\Gamma(q-2)}\|f(s, x(s))-f(s, y(s))\| d s \\
+\frac{1}{48}\left|6 t^{2} T-4 t^{3}-T^{3}\right| \int_{0}^{T} \frac{(T-s)^{q-4}}{\Gamma(q-3)}\|f(s, x(s))-f(s, y(s))\| d s \\
\left.+\frac{1}{48}\left|2 T t^{3}-t^{4}-t T^{3}\right| \int_{0}^{T} \frac{(T-s)^{q-5}}{\Gamma(q-4)}\|f(s, x(s))-f(s, y(s))\| d s\right\}
\end{aligned}
$$


Abstract and Applied Analysis

$$
\begin{aligned}
\leq L\|x-y\| \max _{t \in[0, T]}\{ & \frac{1}{\Gamma(q)} \int_{0}^{t}(t-s)^{q-1} d s+\frac{1}{2 \Gamma(q)} \int_{0}^{T}(T-s)^{q-1} d s \\
& +\frac{|T-2 t|}{4 \Gamma(q-1)} \int_{0}^{T}(T-s)^{q-2} d s+\frac{|t(T-t)|}{4 \Gamma(q-2)} \int_{0}^{T}(T-s)^{q-3} d s \\
& +\frac{1}{48 \Gamma(q-3)}\left|6 t^{2} T-4 t^{3}-T^{3}\right| \int_{0}^{T}(T-s)^{q-4} \Gamma(q-3) d s \\
& \left.+\frac{1}{48}\left|2 T t^{3}-t^{4}-t T^{3}\right| \int_{0}^{T} \frac{(T-s)^{q-5}}{\Gamma(q-4)}\right\} \\
\leq \frac{L T^{q}}{2 \Gamma(q+1)}(3+ & \left.\frac{q}{2}+\frac{q(q-1)\left(5 q^{2}-9 q+46\right)}{384}\right)\|x-y\|=\Delta L\|x-y\|,
\end{aligned}
$$

where we have used (3.22). It follows by the condition (3.21) that $\mathcal{U}$ is a contraction. So, by Banach's contraction mapping principle, problem (1.1) has a unique solution.

Example 3.8. Consider the following antiperiodic fractional boundary value problem:

$$
\begin{gathered}
{ }^{C} D^{q} x(t)=\frac{e^{\left(1-\cos ^{2} x(t)\right)^{2}}\left[4 \sin 2 t+8 \ln \left(17+5 \cos ^{2} x(t)\right)\right]}{\sqrt{(17+\sin x(t))}}, \quad 0<t<1, \\
x(0)=-x(1), \quad x^{\prime}(0)=-x^{\prime}(1), \quad x^{\prime \prime}(0)=-x^{\prime \prime}(1), \\
x^{\prime \prime \prime}(0)=-x^{\prime \prime \prime}(1), \quad x^{i v}(0)=-x^{i v}(1),
\end{gathered}
$$

where $4<q \leq 5$ and $T=1$.

Clearly, $|f(t, x)| \leq L_{1}=e(1+2 \ln 22)$, and the hypothesis of Theorem 3.3 holds. Therefore, the conclusion of Theorem 3.3 applies to problem (3.25).

Example 3.9. Consider the following problem:

$$
\begin{gathered}
{ }^{C} D^{q} x(t)=x\left(a^{2}+x^{3}(t)\right)^{1 / 2}+2\left(1+t^{4}\right)^{3}(1-\cos x(t)), \quad x \neq 0, a>0,0<t<1, \\
x(0)=-x(1), \quad x^{\prime}(0)=-x^{\prime}(1), \quad x^{\prime \prime}(0)=-x^{\prime \prime}(1), \\
x^{\prime \prime \prime}(0)=-x^{\prime \prime \prime}(1), \quad x^{i v}(0)=-x^{i v}(1),
\end{gathered}
$$

where $4<q \leq 5$, and $T=1$.

For sufficiently small $x$ (ignoring $x^{2}$ and higher powers of $x$ ), we have

$$
\left|x\left(a^{2}+x^{3}(t)\right)^{1 / 2}+2\left(1+t^{4}\right)^{3}(1-\cos x(t))\right| \leq a|x|,
$$


where $a \leq \delta$, and (3.10) takes the form

$$
\left(\frac{3}{2 \Gamma(q+1)}+\frac{1}{4 \Gamma(q)}+\frac{1}{16 \Gamma(q-1)}+\frac{1}{48 \Gamma(q-2)}+\frac{5}{768 \Gamma(q-3)}\right) \delta \leq 1
$$

(in particular, for $q=9 / 2, \delta \leq 1920 \sqrt{\pi} / 313$ ). Thus all the assumptions of Theorem 3.4 hold. Consequently, the conclusion of Theorem 3.4 implies that the problem (3.26) has at least one solution

Example 3.10. Consider the following antiperiodic fractional boundary value problem:

$$
\begin{aligned}
{ }^{c} D^{9 / 2} x(t) & =\frac{1}{\sqrt{(t+2025)}}\left(\frac{|x|}{1+|x|}+\tan ^{-1} x\right)+\sin t, \quad t \in[0, \pi], \\
x(0) & =-x(\pi), \quad x^{\prime}(0)=-x^{\prime}(\pi), \quad x^{\prime \prime}(0)=-x^{\prime \prime}(\pi), \\
x^{\prime \prime \prime}(0) & =-x^{\prime \prime \prime}(\pi), \quad x^{(i v)}(0)=-x^{(i v)}(\pi),
\end{aligned}
$$

where $q=9 / 2$, and $T=\pi$. Clearly, $L=2 / 45$ as $|f(t, x)-f(t, y)| \leq 2 / 45|x-y|$. Further,

$$
L \Delta=\frac{L T^{q}}{2 \Gamma(q+1)}\left(3+\frac{q}{2}+\frac{q(q-1)\left(5 q^{2}-9 q+46\right)}{384}\right)=\frac{313 \pi^{4}}{43200}<1
$$

Thus, all the assumptions of by Theorem 3.7 are satisfied. Hence, the fractional boundary value problem (3.30) has a unique solution on $[0, \pi]$.

\section{References}

[1] I. Podlubny, Fractional DifferentialEquations, Academic Press, San Diego, Calif, USA, 1999.

[2] G. M. Zaslavsky, Hamiltonian Chaos and Fractional Dynamics, Oxford University Press, Oxford, UK, 2005.

[3] R. L. Magin, Fractional Calculus in Bioengineering, Begell House Publisher, Connecticut, Conn, USA, 2006.

[4] A. A. Kilbas, H. M. Srivastava, and J. J. Trujillo, Theory and Applications of Fractional Differential Equations, vol. 204 of North-Holland Mathematics Studies, Elsevier Science B.V., Amsterdam, The Netherlands, 2006.

[5] J. Sabatier, O. P. Agrawal, and J. A. T. Machado, Advances in Fractional Calculus: Theoretical Developments and Applications in Physics and Engineering, Springer, Dordrecht, The Netherlands, 2007.

[6] D. Baleanu, K. Diethelm, E. Scalas, and J. J. Trujillo, Fractional calculus models and numerical methods. Series on Complexity, Nonlinearity and Chaos, World Scientific Publishing Co. Pte. Ltd., Hackensack, NJ, USA, 2012.

[7] M. P. Lazarevic and A. M. Spasic, "Finite-time stability analysis of fractional order time-delay systems: Gronwall's approach," Mathematical and Computer Modelling, vol. 49, no. 3-4, pp. 475-481, 2009.

[8] B. Ahmad and J. J. Nieto, "Existence results for a coupled system of nonlinear fractional differential equations with three-point boundary conditions," Computers $\mathcal{E}$ Mathematics with Applications, vol. 58, no. 9, pp. 1838-1843, 2009.

[9] R. P. Agarwal, M. Belmekki, and M. Benchohra, "A survey on semilinear differential equations and inclusions involving Riemann-Liouville fractional derivative," Advances in Difference Equations, vol. 2009, Article ID 981728, 47 pages, 2009. 
[10] V. Gafiychuk and B. Datsko, "Mathematical modeling of different types of instabilities in time fractional reaction-diffusion systems," Computers \& Mathematics with Applications, vol. 59, no. 3, pp. 11011107, 2010.

[11] R. P. Agarwal, V. Lakshmikantham, and J. J. Nieto, “On the concept of solution for fractional differential equations with uncertainty," Nonlinear Analysis. Theory, Methods E Applications, vol. 72, no. 6, pp. 2859-2862, 2010.

[12] B. Ahmad, "Existence of solutions for irregular boundary value problems of nonlinear fractional differential equations," Applied Mathematics Letters, vol. 23, no. 4, pp. 390-394, 2010.

[13] J. J. Nieto, "Maximum principles for fractional differential equations derived from Mittag-Leffler functions," Applied Mathematics Letters, vol. 23, no. 10, pp. 1248-1251, 2010.

[14] D. Baleanu, O. G. Mustafa, and R. P. Agarwal, "An existence result for a superlinear fractional differential equation," Applied Mathematics Letters, vol. 23, no. 9, pp. 1129-1132, 2010.

[15] E. Hernandez, D. O'Regan, and K. Balachandran, "On recent developments in the theory of abstract differential equations with fractional derivatives," Nonlinear Analysis. Theory, Methods \& Applications, vol. 73, no. 10, pp. 3462-3471, 2010.

[16] D. Baleanu, O. G. Mustafa, and D. O'Regan, "A Nagumo-like uniqueness theorem for fractional differential equations," Journal of Physics A, vol. 44, no. 39, Article ID 392003, 6 pages, 2011.

[17] B. Ahmad and J. J. Nieto, "Riemann-Liouville fractional integro-differential equations with fractional nonlocal integral boundary conditions," Boundary Value Problems, vol. 2011, p. 36, 2011.

[18] B. Ahmad, J. J. Nieto, A. Alsaedi, and M. El-Shahed, "A study of nonlinear Langevin equation involving two fractional orders in different intervals," Nonlinear Analysis. Real World Applications, vol. 13, no. 2, pp. 599-606, 2012.

[19] G. Wang, D. Baleanu, and L. Zhang, "Monotone iterative method for a class of nonlinear fractional differential equations," Fractional Calculus and Applied Analysis, vol. 15, no. 2, pp. 244-252, 2012.

[20] A. Aghajani, Y. Jalilian, and J. J. Trujillo, "On the existence of solutions of fractional integro-differential equations," Fractional Calculus and Applied Analysis, vol. 15, no. 1, pp. 44-69, 2012.

[21] B. Ahmad and J. J. Nieto, "Existence of solutions for anti-periodic boundary value problems involving fractional differential equations via Leray-Schauder degree theory," Topological Methods in Nonlinear Analysis, vol. 35, no. 2, pp. 295-304, 2010.

[22] B. Ahmad, "Existence of solutions for fractional differential equations of order $q \in(2,3]$ with antiperiodic boundary conditions," Journal of Applied Mathematics and Computing, vol. 34, no. 1-2, pp. 385-391, 2010.

[23] R. P. Agarwal and B. Ahmad, "Existence theory for anti-periodic boundary value problems of fractional differential equations and inclusions," Computers E Mathematics with Applications, vol. 62, no. 3, pp. 1200-1214, 2011.

[24] B. Ahmad and J. J. Nieto, "Anti-periodic fractional boundary value problems," Computers $\mathcal{E}$ Mathematics with Applications, vol. 62, no. 3, pp. 1150-1156, 2011.

[25] G. Wang, B. Ahmad, and L. Zhang, "Impulsive anti-periodic boundary value problem for nonlinear differential equations of fractional order," Nonlinear Analysis. Theory, Methods $\mathcal{E}$ Applications, vol. 74, no. 3, pp. 792-804, 2011.

[26] J. Cao, Q. Yang, and Z. Huang, "Existence of anti-periodic mild solutions for a class of semilinear fractional differential equations," Communications in Nonlinear Science and Numerical Simulation, vol. 17, no. 1, pp. 277-283, 2012.

[27] B. Ahmad and J. J. Nieto, “Anti-periodic fractional boundary value problems with nonlinear term depending on lower order derivative," Fractional Calculus and Applied Analysis, vol. 15, no. 3, pp. 451462, 2012.

[28] J. X. Sun, Nonlinear Functional Analysis and its Application, Science Press, Beijing, China, 2008.

[29] D. R. Smart, Fixed Point Theorems, Cambridge University Press, London, UK, 1980. 


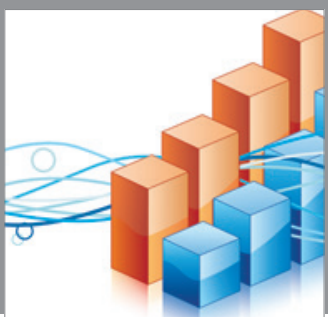

Advances in

Operations Research

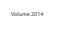

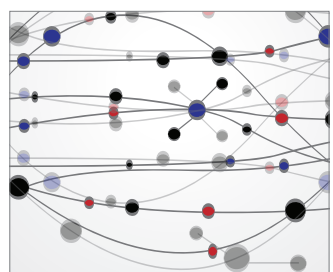

\section{The Scientific} World Journal
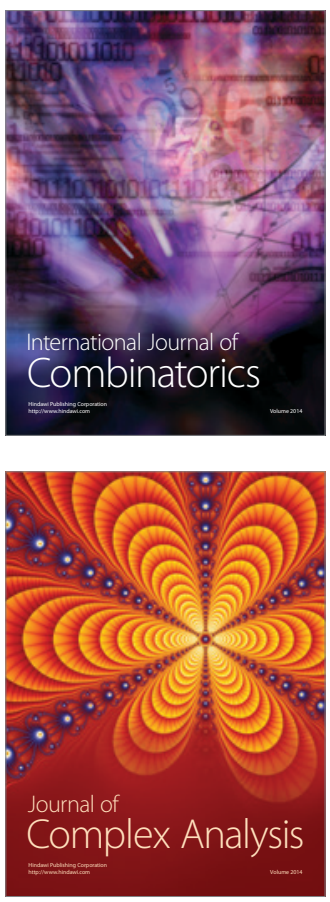

International Journal of

Mathematics and

Mathematical

Sciences
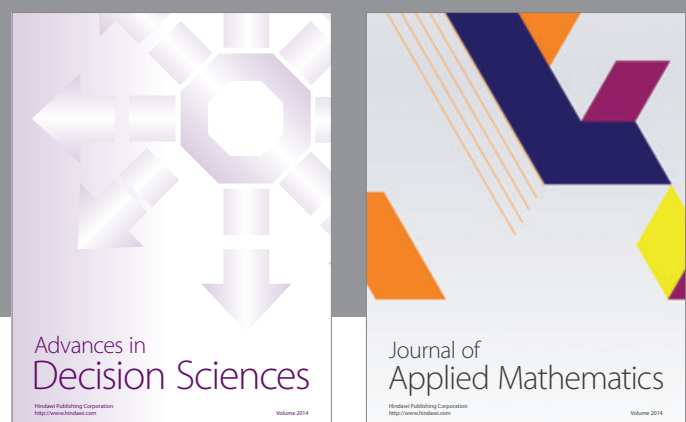

Journal of

Applied Mathematics
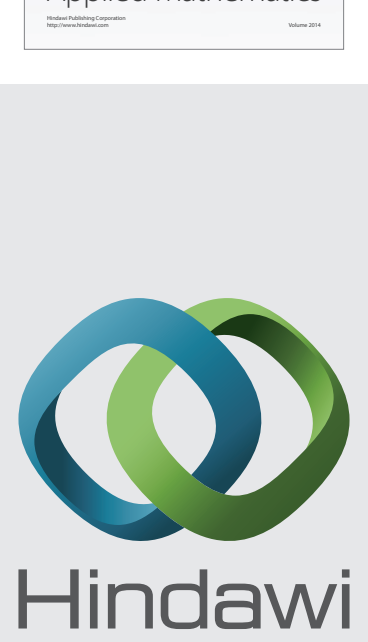

Submit your manuscripts at http://www.hindawi.com
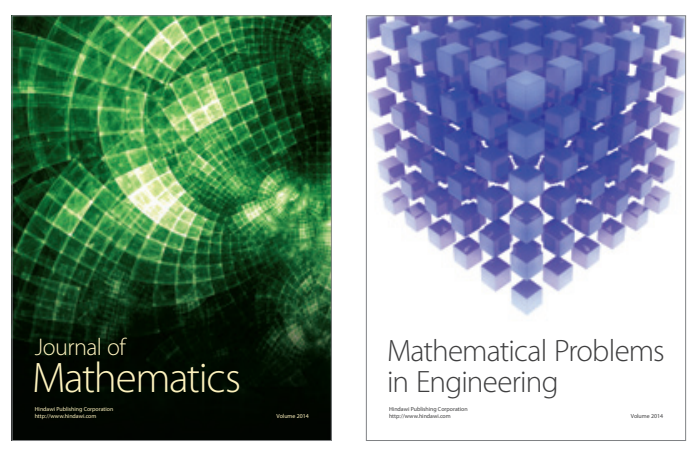

Mathematical Problems in Engineering
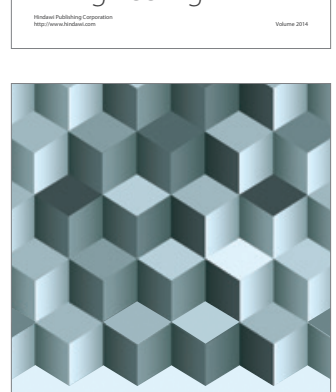

Journal of

Function Spaces
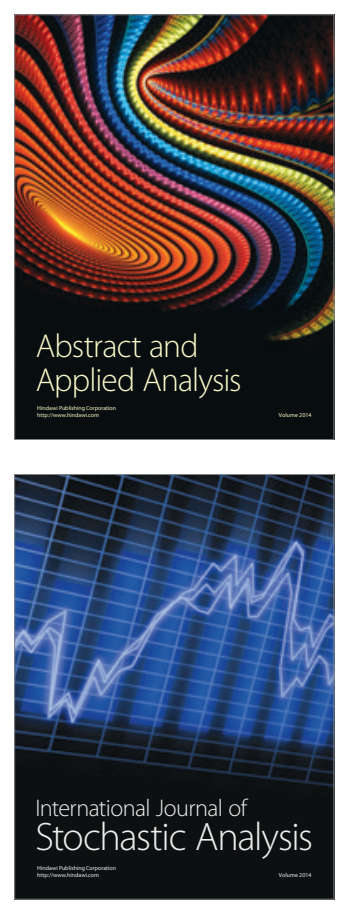

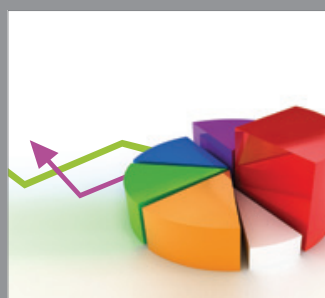

ournal of

Probability and Statistics

Promensencen
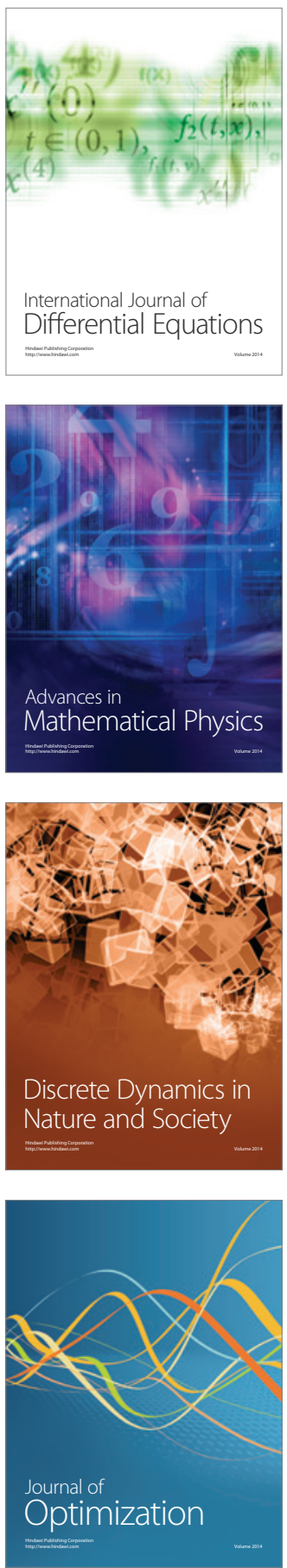